MATHEMATICS OF COMPUTATION

Volume 74, Number 250, Pages 1025-1031

S 0025-5718(04)01674-6

Article electronically published on July 20, 2004

\title{
FIVE CONSECUTIVE POSITIVE ODD NUMBERS, NONE OF WHICH CAN BE EXPRESSED AS A SUM OF TWO PRIME POWERS
}

\author{
YONG-GAO CHEN
}

\begin{abstract}
In this paper, we prove that there is an arithmetic progression of positive odd numbers for each term $M$ of which none of five consecutive odd numbers $M, M-2, M-4, M-6$ and $M-8$ can be expressed in the form $2^{n} \pm p^{\alpha}$, where $p$ is a prime and $n, \alpha$ are nonnegative integers.
\end{abstract}

\section{INTRODUCTION}

By calculation, we find that almost all positive odd numbers can be expressed in the form $2^{n}+p$, where $n$ is a positive integer and $p$ is prime. For example, $5=2+3,7=2+5,9=2+7,11=2^{2}+7,13=2+11,15=2+13$, $17=2^{2}+13$, etc. The first counterexample is 127. In 1934, Romanoff [11 proved that the set of positive odd numbers which can be expressed in the form $2^{n}+p$ has positive asymptotic density in the set of all positive odd numbers, where $n$ is a nonnegative integer and $p$ is prime. For a positive integer $n$ and an integer $a$, let $a(\bmod n)=\{a+n k: k \in \mathbf{Z}\} .\left\{a_{i}\left(\bmod m_{i}\right)\right\}_{i=1}^{k}$ is called a covering system if every integer $b$ satisfies $b \equiv a_{i}\left(\bmod m_{i}\right)$ for at least one value of $i$. By employing a covering system, P. Erdős [8] proved that there is an infinite arithmetic progression of positive odd numbers each of which has no representation of the form $2^{n}+p$. Cohn and Selfridge 7] proved that there exist infinitely many odd numbers which are neither the sum nor the difference of two prime powers. In 3 . Chen proved the following result: the set of positive integers which have no representation of the form $2^{n} \pm p^{a} q^{b}$, where $p, q$ are distinct odd primes and $n, a, b$ are nonnegative integers, has positive lower asymptotic density in the set of all positive odd integers. That is, the lower asymptotic density of the set of positive odd integers $k$ such that $k-2^{n}$ has at least three distinct prime factors for all positive integers $n$ is positive. In 5 Chen showed that the set of positive odd integers $k$ such that $k-2^{n}$ has at least three distinct prime factors for all positive integers $n$ contains an infinite arithmetic progression. For further related information see Chen [4], 6], Guy [9, A19, B21, F13], Jaeschke [10, and Stanton and Williams [12. The following question is a natural one: Are there two consecutive positive odd numbers neither of which can be expressed as a sum of two prime powers?

Received by the editor January 2, 2003 and, in revised form, October 2, 2003.

2000 Mathematics Subject Classification. Primary 11A07, 11B25.

Key words and phrases. Covering systems, odd numbers, sums of prime powers.

Supported by the National Natural Science Foundation of China, Grant No. 10171046 and the Teaching and Research Award Program for Outstanding Young Teachers in Nanjing Normal University. 
In this paper, we show that the answer to the question is affirmative. In fact, we go much further.

Theorem 1. Let $k_{1}, \ldots, k_{s}$ be integers, let $\left\{a_{i j}\left(\bmod m_{i j}\right)\right\}_{j=1}^{t_{i}}(1 \leq i \leq s)$ be $s$ covering systems with $0 \leq a_{i j}<m_{i j}$, and let $p_{i j}$ be primes with $m_{i j}$ the order of $2\left(\bmod p_{i j}\right)\left(1 \leq j \leq t_{i}, 1 \leq i \leq s\right)$ such that if $p_{i j}=p_{u v}$, then

$$
2^{a_{i j}}-k_{i} \equiv 2^{a_{u v}}-k_{u}\left(\bmod p_{i j}\right) .
$$

Then there exists an arithmetic progression of positive odd numbers for each term $M$ of which none of $M+k_{i}(1 \leq i \leq s)$ can be expressed in the form $2^{n} \pm p^{\alpha}$, where $p$ is a prime and $n, \alpha$ are nonnegative integers.

Theorem 2. There exists an arithmetic progression of positive odd numbers for each term $M$ of which none of five consecutive odd numbers $M, M-2, M-4, M-6$ and $M-8$ can be expressed in the form $2^{n} \pm p^{\alpha}$, where $p$ is a prime and $n, \alpha$ are nonnegative integers.

Remark. By the proofs of Theorems 1 and 2, there is an integer $M \leq 2^{2^{253000}}$ such that none of five consecutive odd numbers $M, M-2, M-4, M-6$ and $M-8$ can be expressed in the form $2^{n} \pm p^{\alpha}$. Currently, we cannot give an explicit value of $M$.

\section{Proofs}

Lemma 1. Let $p$ be an odd prime and let $T$ be a positive integer. Then $2^{p^{T}}-1$ has at least $T$ distinct prime factors.

Proof. Let $q_{i}(i=1,2, \ldots, T)$ be primes with

$$
q_{i} \mid \frac{2^{p^{i}}-1}{2^{p^{i-1}}-1} \text {. }
$$

Then $q_{1}, q_{2}, \ldots, q_{T}$ are distinct primes. This completes the proof of Lemma 1 .

Lemma 2. Let $p$ be an odd prime and let $m$ be the order of $2(\bmod p)$. If

$$
2^{m}=1+p^{l} d, \quad p \nmid d,
$$

and $p^{u} \mid 2^{n}-1$ for two integers $n \geq 0$ and $u>0$, then $n=m p^{u-l} v$ for some integer $v$.

Proof. By using induction on $r$, we can prove that

$$
2^{m p^{r}}=1+p^{l+r} d_{r}, \quad p \nmid d_{r}, \quad r=0,1, \ldots
$$

By $p \mid 2^{n}-1$ and $m$ being the order of $2(\bmod p)$, we have $m \mid n$. Let $n=m p^{h} v^{\prime}$, $p \nmid v^{\prime}$. Then

$$
2^{n}=2^{m p^{h} v^{\prime}}=1+p^{l+h} d_{h}^{\prime}, \quad p \nmid d_{h}^{\prime} .
$$

Since $p^{u} \mid 2^{n}-1$, we have $u \leq l+h$. Hence $h \geq u-l$. Let $v=v^{\prime} p^{h-u+l}$. This completes the proof of Lemma 2 .

Lemma 3. Let $p_{1}, \ldots, p_{t}$ be primes such that each prime repeats at most $s$ times. Then there exist $t$ distinct primes $q_{1}, \ldots, q_{t}$ such that

$$
q_{i} \mid 2^{p_{i}^{t+s}}-1, \quad q_{i} \neq p_{j}, \quad \text { for all } i, j .
$$


Proof. For each prime $p$, by Lemma 1 we may take a set $S(p)$ of primes with $|S(p)|=t+s$ such that

$$
q \mid 2^{p^{t+s}}-1
$$

Since there are at most $s$ indexes $i$ with $p_{i}=p$, we may appoint a prime $q_{i} \in$ $S(p) \backslash\left\{p_{1}, \ldots, p_{t}\right\}$ for each $i$ with $p_{i}=p$ such that if $p_{i}=p_{j}=p$, then $q_{i} \neq q_{j}$. If $p_{i} \neq p_{j}$, then, by $q_{i} \in S\left(p_{i}\right)$ and $q_{j} \in S\left(p_{j}\right)$ we have

$$
q_{i}\left|2^{p_{i}^{t+s}}-1, \quad q_{j}\right| 2^{p_{j}^{t+s}}-1 .
$$

Hence $q_{i} \neq q_{j}$. Thus, these $q_{i}$ are distinct such that

$$
q_{i} \mid 2^{p_{i}^{t+s}}-1, \quad q_{i} \neq p_{j}, \quad \text { for all } i, j .
$$

This completes the proof of Lemma 3.

Proof of Theorem 1. If $p_{i u}=p_{i v}$, then, by $m_{i u}$ and $m_{i v}$ being the orders of $2\left(\bmod p_{i u}\right)$ and $2\left(\bmod p_{i v}\right)$, respectively, we have $m_{i u}=m_{i v}$. By

$$
2^{a_{i u}}-k_{i} \equiv 2^{a_{i v}}-k_{i}\left(\bmod p_{i u}\right)
$$

and $m_{i u}$ of the order of $2\left(\bmod p_{i u}\right)$, we have

$$
a_{i v} \equiv a_{i u}\left(\bmod m_{i u}\right) .
$$

Hence $a_{i u}\left(\bmod m_{i u}\right)=a_{i v}\left(\bmod m_{i v}\right)$. Thus, without loss of generality, we may assume that for each $i$, primes $p_{i 1}, \ldots, p_{i t_{i}}$ are distinct. Let $T=s+t_{1}+\cdots+t_{s}$. By Lemma 3 , for each $p_{i j}$, we may appoint a prime $q_{i j}$ such that all primes $q_{i j}(1 \leq$ $\left.j \leq t_{i}, 1 \leq i \leq s\right)$ are distinct,

$$
q_{i j} \mid 2^{p_{i j}^{T}}-1, \quad 1 \leq j \leq t_{i}, 1 \leq i \leq s,
$$

and $q_{i j} \neq p_{u v}$ for all $1 \leq j \leq t_{i}, 1 \leq i \leq s, 1 \leq v \leq t_{u}, 1 \leq u \leq s$. Let $r_{i j}$ be integers such that $0 \leq r_{i j}<p_{i j}$ and

$$
r_{i j} \equiv 2^{a_{i j}}-k_{i}\left(\bmod p_{i j}\right), \quad 1 \leq j \leq t_{i}, 1 \leq i \leq s .
$$

Let

$$
2^{m_{i j}}=1+p_{i j}^{l_{i j}} t_{i j}, \quad p \nmid t_{i j}, \quad 1 \leq j \leq t_{i}, 1 \leq i \leq s,
$$

and $l=\max _{i, j} l_{i j}$. If there exists a nonnegative integer $b \equiv a_{i j}\left(\bmod m_{i j}\right)$ with

$$
p_{i j}^{l+T} \mid 2^{b}-k_{i}-r_{i j},
$$

then let $b_{i j}$ be the least one of such $b$. If there are no such $b$, then let $b_{i j}=a_{i j}$. Let $m$ be a positive integer with

$$
2^{m} \geq \max _{i, j} p_{i j}^{l+T}+\max _{i}\left|k_{i}\right|+1 .
$$

Take an integer $M$ with

$$
\begin{aligned}
& M \equiv r_{i j}\left(\bmod p_{i j}^{l+T}\right) \\
& M \equiv 2^{b_{i j}}-k_{i}\left(\bmod q_{i j}\right), \quad 1 \leq j \leq t_{j}, 1 \leq i \leq s, \\
& M \equiv 1+2^{m}+2^{m+1}\left(\bmod 2^{m+2}\right) .
\end{aligned}
$$


If $p_{i j}=p_{u v}$, then $r_{i j}=r_{u v}$ by the condition. Again, $q_{i j}$ are distinct and each $q_{i j}$ is different from any $p_{u v}$. So such an $M$ exists by the Chinese Remainder Theorem. Now we prove that none of $M+k_{i}(1 \leq i \leq s)$ can be expressed in the form $2^{n} \pm p^{\alpha}$, where $p$ is a prime and $n, \alpha$ are nonnegative integers. In order to prove this, it is enough to show that for each $i$ and any nonnegative integer $n, M+k_{i}-2^{n}$ has at least two distinct positive prime factors. Since $\left\{a_{i j}\left(\bmod m_{i j}\right)\right\}_{j=1}^{t_{i}}$ is a covering system, there exists a $j$ with

$$
n \equiv a_{i j}\left(\bmod m_{i j}\right)
$$

By $(1),(3)$ and $2^{m_{i j}} \equiv 1\left(\bmod p_{i j}\right)$, we have

$$
M+k_{i}-2^{n} \equiv r_{i j}+k_{i}-2^{a_{i j}} \equiv 0\left(\bmod p_{i j}\right) .
$$

Let

$$
M+k_{i}-2^{n}=p_{i j}^{\alpha_{i j}} K_{i j}, \quad p_{i j} \nmid K_{i j}, \alpha_{i j} \geq 1 .
$$

If $\alpha_{i j}<l+T$, then by

$$
\begin{aligned}
\left|M+k_{i}-2^{n}\right| & =\left|1+2^{m}+2^{m+1}+2^{m+2} u+k_{i}-2^{n}\right| \\
& \geq\left|1+2^{m}+2^{m+1}+2^{m+2} u-2^{n}\right|-\left|k_{i}\right| \\
& \geq 2^{m}-1-\left|k_{i}\right| \geq p_{i j}^{l+T},
\end{aligned}
$$

we have $\left|K_{i j}\right|>1$. In this case, $M+k_{i}-2^{n}$ has at least two distinct prime factors. If $\alpha_{i j} \geq l+T$, then $n \equiv a_{i j}\left(\bmod m_{i j}\right)$ and

$$
r_{i j}+k_{i}-2^{n} \equiv M+k_{i}-2^{n} \equiv 0\left(\bmod p_{i j}^{l+T}\right) .
$$

Hence $n \equiv b_{i j}\left(\bmod m_{i j}\right)$ and by $(2)$,

$$
2^{b_{i j}}\left(1-2^{n-b_{i j}}\right) \equiv 2^{b_{i j}}-k_{i}+k_{i}-2^{n} \equiv r_{i j}+k_{i}-2^{n} \equiv 0\left(\bmod p_{i j}^{l+T}\right) .
$$

Thus

$$
p_{i j}^{l+T} \mid 2^{n-b_{i j}}-1
$$

By Lemma 2 we have $n-b_{i j}=m_{i j} p_{i j}^{T} v_{i j}$ for some integer $v_{i j}$. By

$$
q_{i j} \mid 2^{p_{i j}^{T}}-1
$$

we have

$$
q_{i j} \mid 2^{n-b_{i j}}-1
$$

That is,

$$
q_{i j} \mid 2^{n}-2^{b_{i j}}
$$

Hence

$$
M+k_{i}-2^{n} \equiv 2^{b_{i j}}-k_{i}+k_{i}-2^{n} \equiv 2^{b_{i j}}-2^{n} \equiv 0\left(\bmod q_{i j}\right) .
$$

Thus $q_{i j} \mid K_{i j}$ and then $M+k_{i}-2^{n}$ has at least two distinct prime factors. This completes the proof of Theorem 1. 
Proof of Theorem 2. Let $k_{1}=0, k_{2}=-2, k_{3}=-4, k_{4}=-6$ and $k_{5}=-8$. Take

$$
\begin{aligned}
& \left\{a_{1 j}\left(\bmod m_{1 j}\right)\right\}_{j=1}^{8}=\{0(\bmod 2), \quad 3(\bmod 4), 5(\bmod 8), \\
& 9(\bmod 16), \quad 17(\bmod 32), 33(\bmod 64), \\
& 1(\bmod 128), \quad 65(\bmod 128)\}, \\
& \left\{a_{2 j}\left(\bmod m_{2 j}\right)\right\}_{j=1}^{7}=\{1(\bmod 2), \quad 0(\bmod 4), 6(\bmod 8), \\
& 10(\bmod 16), \quad 18(\bmod 32), 34(\bmod 64), \\
& 2(\bmod 64)\} \text {, } \\
& \left\{a_{3 j}\left(\bmod m_{3 j}\right)\right\}_{j=1}^{26}=\{0(\bmod 3), \quad 2(\bmod 4), 3(\bmod 5), \\
& 1(\bmod 10), \quad 4(\bmod 12), 2(\bmod 15), \\
& 1(\bmod 18), \quad 7(\bmod 20), 8(\bmod 24), \\
& 19(\bmod 25), \quad 24(\bmod 25), 11(\bmod 36), \\
& 23(\bmod 36), \quad 25(\bmod 40), 25(\bmod 45), \\
& 40(\bmod 45), \quad 20(\bmod 48), 44(\bmod 48), \\
& 9(\bmod 50), \quad 39(\bmod 50), 37(\bmod 60), \\
& 35(\bmod 72), \quad 4(\bmod 75), 5(\bmod 120) \text {, } \\
& 29(\bmod 150), 215(\bmod 360)\} \text {, } \\
& \left\{a_{4 j}\left(\bmod m_{4 j}\right)\right\}_{j=1}^{9}=\{0(\bmod 2), \quad 1(\bmod 4), 7(\bmod 8), \\
& 11(\bmod 16), \quad 19(\bmod 32), 35(\bmod 64), \\
& 67(\bmod 128), \quad 3(\bmod 256), 131(\bmod 256)\} \\
& \left\{a_{5 j}\left(\bmod m_{5 j}\right)\right\}_{j=1}^{13}=\{1(\bmod 2), \quad 2(\bmod 3), 2(\bmod 5), \\
& 4(\bmod 9), \quad 6(\bmod 10), 6(\bmod 12), \\
& 10(\bmod 18), \quad 0(\bmod 20), 24(\bmod 30), \\
& 34(\bmod 36), \quad 48(\bmod 60), 34(\bmod 90) \\
& 88(\bmod 180)\} \text {. }
\end{aligned}
$$

Noting that $\left\{a_{j}\left(\bmod m_{j}\right)\right\}_{j=1}^{k}$ is a covering system if and only if for every integer $n$ with $0 \leq n<$ l.c.m. $\left\{m_{1}, \ldots, m_{k}\right\}$ there exists a $j$ with $n \equiv a_{j}\left(\bmod m_{j}\right)$, we can verify that $\left\{a_{1 j}\left(\bmod m_{1 j}\right)\right\}_{j=1}^{8},\left\{a_{2 j}\left(\bmod m_{2 j}\right)\right\}_{j=1}^{7},\left\{a_{3 j}\left(\bmod m_{3 j}\right)\right\}_{j=1}^{26}$, $\left\{a_{4 j}\left(\bmod m_{4 j}\right)\right\}_{j=1}^{9}$ and $\left\{a_{5 j}\left(\bmod m_{5 j}\right)\right\}_{j=1}^{13}$ are all covering systems. Now, for every $a_{i j}\left(\bmod m_{i j}\right)$ we appoint a prime $p_{i j}$ such that $m_{i j}$ is the order of $2\left(\bmod p_{i j}\right)$ and if $p_{i j}=p_{u v}$, then

$$
2^{a_{i j}}-k_{i} \equiv 2^{a_{u v}}-k_{u}\left(\bmod p_{i j}\right) .
$$

Case 1. Let $p_{11}=p_{21}=p_{41}=p_{51}=3$. Then

$$
2^{0}-0 \equiv 2^{1}-(-2) \equiv 2^{0}-(-6) \equiv 2^{1}-(-8)(\bmod 3) .
$$

Case 2. Let $p_{12}=p_{22}=p_{32}=p_{42}=5$. Then

$$
2^{3}-0 \equiv 2^{0}-(-2) \equiv 2^{2}-(-4) \equiv 2^{1}-(-6)(\bmod 5) .
$$


Case 3. Let

$$
\begin{gathered}
p_{13}=p_{23}=p_{43}=17, \quad p_{14}=p_{24}=p_{44}=257 \\
p_{15}=p_{25}=p_{45}=65537, \quad p_{16}=p_{26}=p_{46}=641, \quad p_{27}=6700417 .
\end{gathered}
$$

Note that both Fermat numbers $F_{6}$ and $F_{7}$ are composite, let $p_{18}=p_{47}, p_{17}$ be two distinct prime divisors of $2^{64}+1$, and let $p_{48}, p_{49}$ be two distinct prime divisors of $2^{128}+1$. Then (4) follows from the following fact:

$$
2^{2^{k}+1}-0 \equiv 2^{2^{k}+2}-(-2) \equiv 2^{2^{k}+3}-(-6)\left(\bmod 2^{2^{k}}+1\right) .
$$

Case 4. Let

$$
\begin{gathered}
p_{31}=p_{52}=7, \quad p_{33}=p_{53}=31 \\
p_{34}=p_{55}=11, \quad p_{35}=p_{56}=13 \\
p_{37}=p_{57}=19, \quad p_{38}=p_{58}=41 \\
p_{3(12)}=p_{5(10)}=109, \quad p_{3(13)}=37
\end{gathered}
$$

Then

$$
\begin{array}{cc}
2^{0}-(-4) \equiv 2^{2}-(-8)(\bmod 7), & 2^{3}-(-4) \equiv 2^{2}-(-8)(\bmod 31) \\
2^{1}-(-4) \equiv 2^{6}-(-8)(\bmod 11), & 2^{4}-(-4) \equiv 2^{6}-(-8)(\bmod 13), \\
2^{1}-(-4) \equiv 2^{10}-(-8)(\bmod 19), & 2^{7}-(-4) \equiv 2^{0}-(-8)(\bmod 41), \\
2^{11}-(-4) \equiv 2^{34}-(-8)(\bmod 109)
\end{array}
$$

Case 5. Each of 25, 45, 48, 50, 60 is the order of 2 modulus two distinct primes. These primes are 601,$1801 ; 631,23311 ; 97,673 ; 251,4051 ; 61,1321$, respectively. If $m>1$ and $m \neq 6$, then there exists at least one prime $p$ with $m$ the order of $2(\bmod p)\left(\right.$ see [1], [2], [13]). Thus we may appoint a prime $p_{i j}$ for each of the remaining $a_{i j}\left(\bmod m_{i j}\right)$. Now, Theorem 2 follows from Theorem 1.

\section{ACKNOWLEDGMENT}

I am grateful to the referee for his/her suggestion.

\section{REFERENCES}

[1] A. S. Bang, Taltheoretiske Unders $\phi$ gelser, Tidsskrift for Mat. (5), 4(1886), 70-80, 130-137.

[2] G. D. Birkhoff and H. S. Vandiver, On the integral divisors of $a^{n}-b^{n}$, Ann. Math. 5(1904), 173-180.

[3] Y. G. Chen, On integers of the form $2^{n} \pm p_{1}^{\alpha_{1}} \cdots p_{r}^{\alpha_{r}}$, Proc. Amer. Math. Soc. 128(2000), 1613-1616. MR 2000j:11006

[4] Y. G. Chen, On integers of the form $k 2^{n}+1$, Proc. Amer. Math. Soc. 129(2001), 355-361. MR 2003a:11004

[5] Y. G. Chen, On integers of the forms $k-2^{n}$ and $k 2^{n}+1$, J. Number Theory 89(2001), 121-125. MR 2002b:11020

[6] Y. G. Chen, On integers of the forms $k^{r}-2^{n}$ and $k^{r} 2^{n}+1$, J. Number Theory 98(2003), 310-319. MR 2003m:11004

[7] F. Cohen and J. L. Selfridge, Not every number is the sum or difference of two prime powers, Math. Comput. 29(1975), 79-81. MR 51:12758

[8] P. Erdős, On integers of the form $2^{r}+p$ and some related problems, Summa Brasil. Math. 2(1950), 113-123. MR 13:437i

[9] R. K. Guy, "Unsolved problems in number theory," 2nd ed., Springer, New York, 1994. MR 96e: 11002

[10] G. Jaeschke, On the smallest $k$ such that all $k \cdot 2^{N}+1$ are composite, Math. Comput. 40(1983), 381-384; corrigendum, Math. Comput. 45(1985) 637. MR 84k:10006: MR 87b:11009 
[11] N. P. Romanoff, Über einige Sätze der additiven Zahlentheorie, Math. Ann. 57(1934), 668678.

[12] R. G. Stanton and H. C. Williams, Further results on covering of the integer $1+k 2^{n}$ by primes, in "Combinatorial Math. VIII," Lecture Notes in Math. 884, Springer-Verlag, Berlin/New York, 1980, 107-114. MR 84j:10009

[13] K. Zsigmondy, Zur Theorie der Potenzreste, Monatsh. Math. 3(1892), 265-284.

Department of Mathematics, Nanjing Normal University, Nanjing 210097, Peoples Republic of China

E-mail address: ygchen@pine.njnu.edu.cn 